\title{
Correction to: IgY-Technology: Production and Application of Egg Yolk Antibodies
}

\author{
Xiao-Ying Zhang, Ricardo S. Vieira-Pires, Patricia M. Morgan, \\ and Rüdiger Schade
}

\section{Correction to: \\ X.-Y. Zhang et al. (eds.), IgY-Technology: \\ Production and Application of Egg Yolk Antibodies, https://doi.org/10.1007/978-3-030-72688-1}

The original version of the book was inadvertently published with only one of the three affiliations of the volume editor and chapter author Xiao-Ying Zhang.

The correct affiliations are given here.

College of Biological Science and Engineering, Shaanxi University of Technology, Hanzhong, China;

Ontario Veterinary College, University of Guelph, Guelph, ON, Canada;

CBMA, University of Minho, Braga, Portugal

zhxying@snut.edu.cn; xzhang67@uoguelph.ca; zhang@bio.uminho.pt

This has now been corrected in the chapters.

\footnotetext{
The updated versions of the chapters can be found at https://doi.org/10.1007/978-3-030-72688-1_1 https://doi.org/10.1007/978-3-030-72688-1_2 https://doi.org/10.1007/978-3-030-72688-1_4 https://doi.org/10.1007/978-3-030-72688-1_5 https://doi.org/10.1007/978-3-030-72688-1_6 https://doi.org/10.1007/978-3-030-72688-1_7 https://doi.org/10.1007/978-3-030-72688-1_10 https://doi.org/10.1007/978-3-030-72688-1_11 https://doi.org/10.1007/978-3-030-72688-1_12 https://doi.org/10.1007/978-3-030-72688-1_13 https://doi.org/10.1007/978-3-030-72688-1_15 https://doi.org/10.1007/978-3-030-72688-1_18
} 